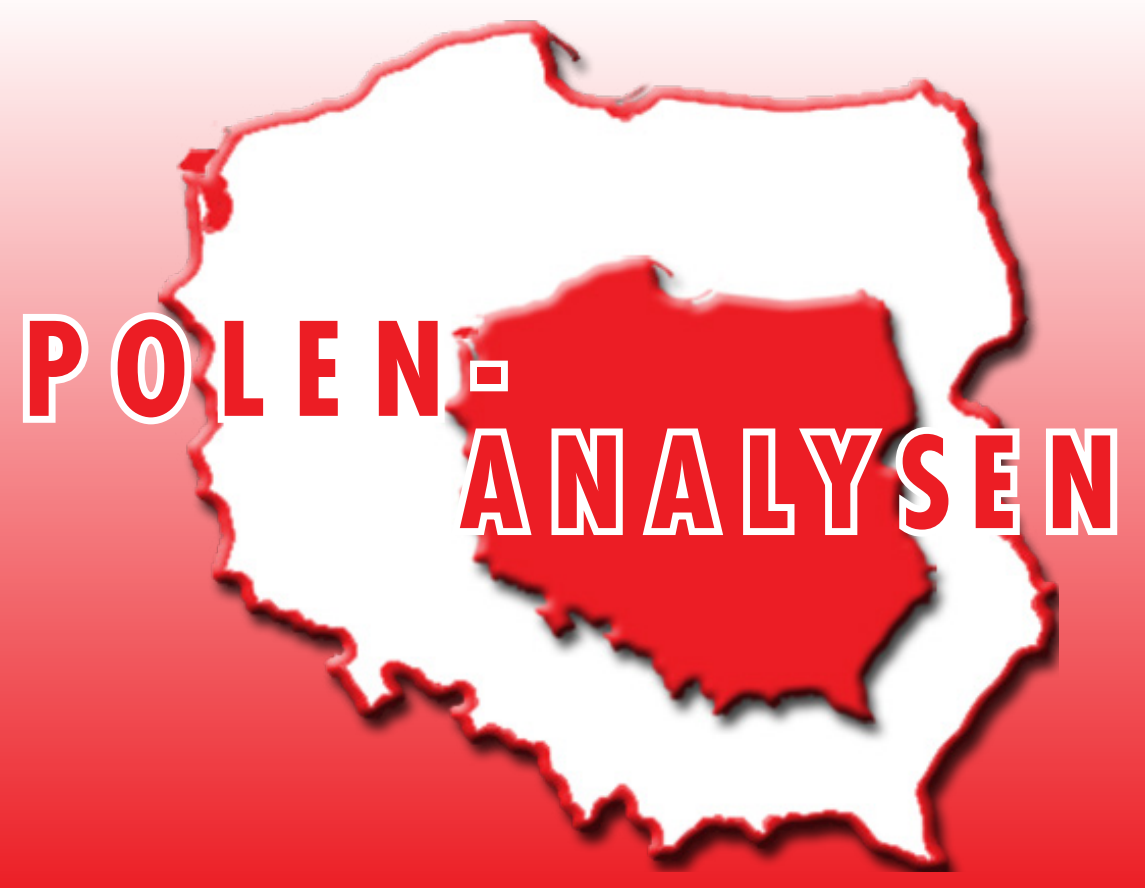

www.laender-analysen.de/polen

\title{
DIE ABTREIBUNGSDEBATTE
}

ANALYSE

»Schwarzer Protest« - in Richtung eines neuen »Kompromisses« beim Abtreibungsrecht? 2 Małgorzata Druciarek, Warschau

- TABELLEN UND GRAFIKEN ZUM TEXT

Umfragen zur Abtreibungsdebatte

CHRONIK

1. - 14. November 2016

Herausgegeben mit finanzieller Unterstützung

der Stiftung für deutsch-polnische Zusammenarbeit

FUNDACJA WSPÓŁPRACY

POLSKO-NIEMIECKIEJ

STIFTUNG

FÜR DEUTSCH-POLNISCHE

ZUSAMMENARBEIT

\section{DEUTSCHES POLEN INSTITUT}

- Forschungsstelle Osteuropa an der Universität Bremen
Deutsche Gesellschaft

DGO für Osteuropakunde e.V. 


\title{
"Schwarzer Protest« - in Richtung eines neuen »Kompromisses« beim Abtreibungsrecht?
}

\author{
Małgorzata Druciarek, Warschau
}

\section{Zusammenfassung}

Der 3. Oktober 2016 war in Polen der Tag des »schwarzen Protestes«, eines »Landesweiten Streiks der Frauen«, der von Ereignissen in Island vor 40 Jahren inspiriert worden war. Am 24. Oktober 1975 hatten dort 90 Prozent der Frauen die Arbeit niedergelegt. Dieser eine Tag hatte eine weitreichende politische Entwicklung in Gang gesetzt. In Polen lehnte der Sejm drei Tage nach den Protesten den Bürgergesetzesentwurf zu einem vollständigen Abtreibungsverbot ab. Gleichzeitig kündigte die Regierung von Recht und Gerechtigkeit (Prawo i Sprawiedliwość - PiS) Arbeiten an einem eigenen, großzügig angelegten Programm zum Schutz des ungeborenen Lebens an. In welche Richtung weisen die legislativen Arbeiten der Regierung? Was denkt die polnische Gesellschaft über die von konservativen Politikern vorgeschlagenen Veränderungen? Erwartet uns ein neuer »Kompromiss« im Abtreibungsrecht?

\) $\mathrm{C}_{\text {rung von } \text { Recht und Gerechtigkeit aufgehalten«, }}^{\text {ine gentiche Mobilisierung hat die Regie- }}$ »Radikale Umkehr in den Entscheidungen der Regierungspartei nach massenhaften Protesten von Frauen«, "Nach dem schwarzen Montag zieht die Regierung den Gesetzesentwurf zum Abtreibungsverbot in Polen zurück « - so stellte die Presse in aller Welt die Ereignisse vom 3. Oktober 2016 vor. Die ausländischen Medien verbreiteten Fotos von Tausenden schwarz gekleideter polnischer Frauen, denen es als ersten nach dem Regierungswechsel im Herbst 2015 gelang, sich dem Eifer der konservativen Abgeordneten wirksam entgegenzustellen. In Westeuropa, Kanada und den Vereinigten Staaten war man von dem spektakulären Erfolg der Polinnen beeindruckt. Der 3. Oktober war der Tag des »schwarzen Protestes«, eines "Landesweiten Streiks der Frauen«, der von Ereignissen in Island vor 40 Jahren inspiriert worden war. Am 24. Oktober 1975 hatten dort 90 Prozent der Frauen die Arbeit niedergelegt; auch diejenigen, die als Hausfrauen tätig waren, hörten auf zu kochen, zu waschen und sich um die Kinder zu kümmern. Dieser eine Tag hatte eine Revolution in Gang gesetzt. In Polen lehnte der Sejm drei Tage nach den Protesten den Bürgergesetzesentwurf zu einem vollständigen Abtreibungsverbot ab. Gleichzeitig kündigte die PiS-Regierung Arbeiten an einem eigenen, großzügig angelegten Programm zum Schutz des ungeborenen Lebens an. In welche Richtung weisen die legislativen Arbeiten der Regierung? Was denkt die polnische Gesellschaft über die von den konservativen Politikern vorgeschlagenen Veränderungen? Erwartet uns ein neuer »Kompromiss« im Abtreibungsrecht?

\section{Der "Abtreibungskompromiss"}

Der politische Umbruch im Jahr 1989 brachte eine deutliche Beschränkung der bis dahin sehr liberalen Abtrei- bungsgesetzgebung in Polen mit sich. Dabei handelte es sich um eine Forderung der katholischen Kirche, die von einem Teil der Politiker des Solidarność-Lagers unterstützt wurde. Vor dem Hintergrund, dass in keinem anderen postsozialistischen Land die Gesetzgebung zur Abtreibung nach 1989 eingeschränkt wurde, ist es bemerkenswert, auf welche Weise die Kirche ihr Ziel erreichte. In der Frage der Abtreibung gelang es ihr, viele Politiker zu überzeugen, und das nicht nur, weil sie Katholiken waren. In den 1990er Jahren trat die katholische Kirche als einzige politische Siegerin in Erscheinung. Der Kirchenhierarchie gelang es, eine starke Position in der Öffentlichkeit einzunehmen und die Richtung politischer Veränderungen mitzubestimmen. Die Verabschiedung des Gesetzes über den Schutz des ungeborenen Lebens im Jahr 1993 war nur eines der Beispiele dafür, wie die Kirche Einfluss auf die Gesetzgebung nahm. Des Weiteren hatte die Tatsache, dass die politische Position der Frauen nach der Transformation schwach war, Einfluss auf die damals getroffenen Entscheidungen. Obwohl Frauen Aktivistinnen der demokratischen Opposition gewesen waren, bewirkte das damals nur schwach ausgeprägte feministische Denken, dass sie ihre Interessen nicht in Geschlechterkategorien zum Ausdruck brachten. Im Ergebnis trat eine Verschlechterung der Situation der Frauen nach 1989 ein, und zwar nicht nur im Bereich der Gesetzgebung zum Schwangerschaftsabbruch, sondern auch was die Präsenz der Frauen auf der politischen Bühne oder ihre Situation auf dem Arbeitsmarkt betraf.

Das Gesetz, das den Zugang zum Schwangerschaftsabbruch in Polen reguliert, ist eines der restriktivsten in Europa. Nur in Irland und Malta, den beiden anderen Ländern, die unter dem starken Einfluss der katholischen Kirche stehen, gelten ähnlich restriktive Gesetze. Nach dem "Gesetz über Familienplanung, den Schutz 
des menschlichen Fötus und die Bedingungen für die Zulässigkeit eines Schwangerschaftsabbruchs« vom 7. Januar 1993 ist Abtreibung in Polen nur in drei Fällen erlaubt. Erstens, wenn die Schwangerschaft eine Gefahr für das Leben oder die Gesundheit der Schwangeren darstellt, zweitens im Falle einer schweren und irreversiblen Behinderung des Fötus oder einer unheilbaren Krankheit, die sein Leben bedroht, und drittens, wenn die Schwangerschaft infolge einer Straftat eintrat. Der Kirchenhierarchie gelang es nicht nur, auf die Gestalt der Gesetzgebung Einfluss zu nehmen, sondern auch auf den Diskurs über das Antiabtreibungsgesetz. Eingeführt wurde der Begriff des »empfangenen Kindes« als Rechtssubjekt, dem Schutz und Fürsorge zusteht. Die rechtlichen Änderungen in diesem Bereich wurden als Geste der Befreiung von den sowjetischen Einflüssen dargestellt. Es kam zu dem sogenannten Kompromiss, der in der Realität aber kein Kompromiss zwischen unterschiedlichen Interessensgruppen war. Vielmehr war es ein Vertrag zwischen den Kirchenoberen und konservativen Politikern, eine Lösung, die eine einflussreiche Minderheit der ganzen Gesellschaft auferlegte, die damals aber deutlich liberaler eingestellt war. In den folgenden Jahren betrieb die Kirche die Stigmatisierung von Abtreibungen, Frauen, die sie durchführen ließen, und Ärzten, die sie ausführten. Das außergewöhnlich restriktive Gesetz, die Stigmatisierung des umgebenden Milieus und die fehlende Regulierung in Form einer Gewissensklausel (was von den Ärzten genutzt wurde, um eine Abtreibung abzulehnen) hatten zur Folge, dass es praktisch keinen Zugang zu legalen Eingriffen gab. Unterschiedlichen Schätzungen zufolge lassen bis zu 100.000 Polinnen jährlich eine Abtreibung in Privatkliniken durchführen, indem sie aus dem Ausland importierte Pillen einnehmen oder in Länder fahren, in denen der Abbruch legal und sicher durchgeführt wird. Zweifellos ist der Zugang zu einem sicheren Abtreibungsverfahren von sozioökonomischen Faktoren bestimmt und teilt die Gesellschaft eindeutig in diejenigen, die sich eine echte Wahl in ihren Angelegenheiten der Fortpflanzung leisten können, und die, die keine Wahl haben.

\section{Die neue Regierung und der neue Krieg um Werte}

Betrachtet man Meinungsumfragen zum Verhältnis der Bürgerinnen und Bürger zum Abtreibungsgesetz, kann man zu dem Schluss kommen, dass sich ein deutlicher Teil der Gesellschaft in den letzten zwei Jahrzehnten an das geltende Gesetz gewöhnt hat. Nach einer Umfrage des renommierten Meinungsforschungsinstituts CBOS im März 2016 spricht sich die Mehrheit der Polinnen und Polen für die Beibehaltung des »Abtreibungskompromisses« aus (siehe Tabelle 1 auf Seite 8). Das bedeutet, dass die Befragten mehrheitlich der Meinung sind, dass ein legaler Schwangerschaftsabbruch in den drei Fällen des geltenden Gesetzes zulässig sein sollte: Wenn Gefahr für das Leben der Mutter besteht (80 Prozent) bzw. für ihre Gesundheit (71 Prozent) und wenn die Schwangerschaft infolge einer Straftat eintritt (73 Prozent). Der dritte Fall, das heißt die Zulässigkeit des Schwangerschaftsabbruchs, wenn pränatale Untersuchungen eine dauerhafte Schädigung des Fötus zeigen, erlangte mit 53 Prozent die relativ geringste gesellschaftliche Unterstützung. Am wenigsten akzeptieren die Polen die Zulässigkeit von Abtreibungen aufgrund der schlechten materiellen Situation der Mutter (75 Prozent sind der Ansicht, dass in diesem Fall eine Abtreibung nicht zulässig sein sollte), wenn ihre persönliche Situation schwierig ist (75 Prozent) oder auch, wenn die betreffende Frau kein Kind haben möchte (76 Prozent). Seit dem Jahr 2012 ist die Einstellung der Polen gegenüber dem Schwangerschaftsabbruch aus sozioökonomischen Gründen auf ähnlichem Niveau geblieben. Hier befürworten 14 Prozent der Befragten die Zulässigkeit einer Abtreibung (2012: 16 Prozent). Der Faktor, der die Haltung zum Schwangerschaftsabbruch am stärksten beeinflusst, ist die praktizierte Religiosität.

Der »Abtreibungskompromiss« wurde zum verbindlichen Status quo, den Politiker jahrelang aus Angst vor der Reaktion der Gesellschaft sowie der Kirche nicht $\mathrm{zu}$ ändern wagten. Im Ergebnis erwog bis zum Jahr 2016 keine Regierung ernsthaft eine rechtliche Reform des Zugangs zum Schwangerschaftsabbruch in Polen. Nachdem im Herbst 2015 PiS an die Macht gekommen war, wurde klar, dass der Einfluss der katholischen Kirchenhierarchie auf das politische Leben stärker und gleichzeitig sichtbarer werden wird. Im Parlamentswahlkampf hielt sich die Kirche von einem aktiven Engagement zurück. Neue Wähler zu gewinnen, insbesondere diejenigen, die von der Vorgängerregierung enttäuscht waren, war die Schlüsselaufgabe der Partei von Jarosław Kaczyński. Daher waren die wesentlichen Wahlversprechen im sozioökonomischen Bereich angesiedelt. Dennoch hatte die Unterstützung der Kirche weiter Schlüsselbedeutung für die Sicherstellung der Loyalität der Stammwählerschaft von PiS. Sowohl die Kirchenhierarchie als auch die traditionelle, religiös-konservative Wählerschaft von $P i S$ wussten, dass weltanschauliche Themen auf die politische Agenda zurückkehren würden, wenn PiS die Wahlen gewinnt. Dazu ist es auch gekommen.

Im Juli 2016 wurde die Bürgergesetzesinitiative "Abtreibungstopp" (Stop Aborcji) im Sejm eingereicht. Sie war von der ultrakonservativen Organisation Ordo Iuris vorbereitet worden und beinhaltete ein komplettes Abtreibungsverbot in Polen. Dem Komitee der Geset- 
zesinitiative war es gelungen, knapp 500.000 Unterschriften für den Entwurf zu sammeln. Worin hätten die rechtlichen und sozialen Konsequenzen des Gesetzes bestanden? Die Rechtswissenschaftlerin Monika Płatek stellt in ihrer Beurteilung des Gesetzesentwurfs fest: "[...] unter dem Vorwand eingebracht, Leben zu retten, zielt der Entwurf auf die Würde des Menschen, die Gesundheit der Frauen und der Föten in der pränatalen Phase sowie auf ihr Leben.« Der Entwurf verbietet den Schwangerschaftsabbruch sogar in Fällen einer Vergewaltigung, wenn die Gesundheit und das Leben der Frau gefährdet sind sowie wenn der Fötus ernsthaft geschädigt ist. Płatek weist darauf hin, dass das Projekt von Ordo Iuris den Schutz des Lebens und der Gesundheit auf das Strafrecht stützt. Dies widerspricht dem Grundsatz, dass das Strafrecht im Gesetzgebungsprozess die Rolle der Ultima Ratio spielt, das heißt ein Instrument sein sollte, dass das menschliche Verhalten allein dann regelt, wenn keine anderen Rechtsbereiche mehr anwendbar sind. Der Gesetzesentwurf kriminalisiert ebenfalls Fehlgeburten, indem er sie zum Gegenstand staatsanwaltlicher Untersuchung ihrer Art und Weise macht.

Parallel zur Unterschriftensammlung für das Gesetzesprojekt "Abtreibungsstopp « wurden Unterschriften für eine weitere Bürgergesetzesinitiative gesammelt, die den Schwangerschaftsabbruch in Polen erlauben sollte. Der Entwurf, der vom Komitee der Gesetzesinitiative "Retten wir die Frauen!« (Ratujmy Kobiety) eingebracht wurde, räumt die Möglichkeit des legalen und sicheren Schwangerschaftsabbruchs ein, garantiert den Frauen während der Schwangerschaft vollständige gesundheitliche Betreuung, gibt Zugang zu modernen Verhütungsmethoden und fordert einen verbindlichen Sexualkundeunterricht in den Schulen. Mit anderen Worten, der Gesetzesvorschlag würde die polnische Gesetzgebung im Bereich der Gesetzgebung zur Fortpflanzung an die Standards der Mehrheit der europäischen Länder annähern. Die Unterschriftensammlung für beide Gesetzesinitiativen, insbesondere aber für die von Ordo Iuris, die in den Medien eindeutig stärker präsent war, löste eine öffentliche Debatte aus, wie sie seit langem in Polen nicht stattgefunden hatte. Eine Debatte, die unter Politikern, Kirchenoberen, Experten, aber auch gewöhnlichen Bürgern geführt wird und die ohne Übertreibung als Anfang eines neuen Krieges um Werte bezeichnet werden kann.

Die Annahme einer der beiden Gesetzesvorschläge würde den »Abtreibungskompromiss « aufheben, der das Land seit 1993 regiert. Beide Entwürfe wurden im Sejm eingereicht, und das Gesetzgebungsprozedere wurde am selben Tag, dem 23. September 2016, eröffnet. Trotz des Versprechens der Regierung, dass keine Bürgergesetzesinitiative von den Abgeordneten in der ersten Lesung abgelehnt werden würde, wurde nur der Vorschlag "Abtreibungsstopp« zur weiteren Bearbeitung an den Ausschuss für Gerechtigkeit und Menschenrechte weitergeleitet. Der Entwurf »Retten wir die Frauen!« wurde in erster Lesung abgelehnt. PiS zeigte, dass sie zu einer Änderung des "Abtreibungskompromisses« bereit ist.

\section{Der "schwarze Protest" - ein zivil- gesellschaftlicher Erfolg, aber auch ein politischer?}

Am 3. Oktober 2016 gingen die Frauen in Polen nicht nur zum ersten Mal massenhaft auf die Straße, um zur Frage des Schwangerschaftsabbruchs zu protestieren. Der "schwarze Montag" war auch die erste Massenmobilisierung der polnischen Frauen zur Verteidigung ihrer Rechte. Keine Kundgebung und Demonstration hatte bisher so viele Teilnehmerinnen auf den Plan gerufen. Dem »Landesweiten Streik der Frauen«, der zunächst eine vor allem mit Hilfe der sozialen Medien angekündigte Initiative war, wurden nicht viele Chancen in Aussicht gestellt. Sein Erfolg überstieg die Erwartungen der Organisatorinnen selbst, und zwar nicht nur, was die Anzahl der streikenden Frauen betraf. Informationen des Hauptkommandanten der Polizei zufolge, nahmen an den »schwarzen Protesten« zirka 98.000 Personen teil. Anders als viele erwartet hatten, beschränkte sich der Protest nicht auf die großen Städte - die Initiative "Landesweiter Streik der Frauen« spricht von Protesten in 118 Städten Polens. Zusätzlich kam es im Ausland zu über 50 Solidaritätskundgebungen. Allein das Ausmaß des Protestes zeigt eindeutig, dass es sich hier um einen überragenden Erfolg der Frauen in Polen handelt. Von Bedeutung ist dabei auch, dass Frauen (sowie auch Männer und Kinder) in verschiedenen Regionen Polens auf die Straße gingen, Frauen, die sich in Alter, Ausbildung, sozioökonomischem Status und Weltanschauungen unterschieden. Die Massenproteste der Frauen erhielten bereits in der Vorbereitungsphase große Unterstützung vonseiten der Gesellschaft. Nach Umfragen des Meinungsforschungsinstituts IPSOS vom 28. bis 30. September unterstützte die Mehrheit der Polinnen (55 Prozent) und fast die Hälfte der Polen (45 Prozent) den Aufruhr der Frauen. Gegner des Streiks waren 14 Prozent der Befragten.

Der "schwarze Protest" schlug sich unter anderem auf die Ergebnisse der Meinungsumfragen zu der Zulässigkeit von Abtreibungen nieder. Auf dem Höhepunkt der Vorbereitungen der Demonstrationen gegen die Verschärfung des Abtreibungsgesetzes beauftragte das Portal OKO.press das Institut IPSOS mit der Untersuchung der Einstellung der Polinnen und Polen gegenüber den aktuellen Bestimmungen zum Schwangerschaftsabbruch. Über ein Drittel der Befragten sprach sich für 
die Liberalisierung des geltenden Gesetzes aus, während es in den Umfragen in diesem Jahr vor den Protesten nicht mehr als 30 Prozent waren. OKO.press fragte die Polen unter anderem, ob sie für die Liberalisierung des Abtreibungsgesetzes und die Zulässigkeit von Schwangerschaftsabbrüchen im Falle einer schwierigen Situation der betreffenden Frau seien. Dafür sprachen sich 39 Prozent der Polinnen und 35 Prozent der Polen aus. Für die Liberalisierung des geltenden Gesetzes sprachen sich die Einwohner von Großstädten (über 500.000 Einwohner) vor allem mit Berufsausbildung (43 Prozent) und mittlerem Ausbildungsgrad (37 Prozent) aus. Gleichzeitig beeinflussten die Arbeiten am Gesetzesprojekt von Ordo Iuris und die Proteste der Frauen aber nicht den Anteil derjenigen, die die Verschärfung des Abtreibungsgesetzes befürworteten. Unverändert lag er bei 10 bis 12 Prozent. Die Unterstützung des vollständigen Abtreibungsverbots ist in der Mehrheit der Altersgruppen ähnlich niedrig, allerdings etwas höher bei den jüngsten Befragten (18-29 Jahre). Dies bestätigt die von vielen Experten vertretene These von der Radikalisierung junger Polen. Auch die neuesten Untersuchungen von CBOS zeigen deutlich, dass die Debatte um die beiden Gesetzesentwürfe und die Massenproteste der Frauen die Einstellungen der Polen zur Abtreibung beeinflusst haben (siehe Tabelle 1 auf Seite 8). Beide Faktoren bewirkten vor allem eine Befestigung der Ansichten derjenigen, die Abtreibung für zulässig halten - die Unterstützung von Schwangerschaftsabbrüchen in den drei gesetzlich verankerten Fällen wird entschiedener als vorher zum Ausdruck gebracht. Im Vergleich zu den vorangegangenen Untersuchungen vom März 2016 stieg die Zustimmung zum Schwangerschaftsabbruch fast in jeder Kategorie. Wenn der Anstieg auch nicht spektakulär ist, zeigt sich bereits ein gewisser Trend. Feststellbar ist außerdem ein geringer Anstieg des Anteils derjenigen, die Abtreibung im Falle einer schwierigen materiellen oder persönlichen Situation der Frau befürworten - unter solchen Umständen akzeptiert jeder fünfte Befragte einen Schwangerschaftsabbruch. Während die Mehrheit der Polen sich für die Beibehaltung des "Abtreibungskompromisses» ausspricht (62 Prozent), befürwortet fast jeder vierte Befragte die Liberalisierung des geltenden Rechts. Weitere Untersuchungen werden zeigen, ob man von einer beständig größer werdenden Tolerierung von Schwangerschaftsabbrüchen in anderen Fällen als den im aktuellen Gesetz angeführten sprechen kann.

Der "schwarze Montag" war in vielerlei Hinsicht außergewöhnlich. Agata Chełstowska stellte im Internetmagazin "Codziennik Feministyczny « fest, dass sich im Kontext des Streiks plötzlich ein Raum eröffnet hat, über die eigenen Emotionen zu sprechen - starke Emotionen, die der Gesetzesentwurf zu einem vollständigen
Abtreibungsverbot in Polen bei Tausenden von Frauen ausgelöst hatte. Dies waren Emotionen, die die Aktivistinnen der Frauenbewegung bisher nicht haben zeigen dürfen. Bisher waren das kultivierte Gespräch und stichhaltige Argumente das Wichtigste. Dieses Mal jedoch sei bei den Frauen etwas geplatzt. Ein Umbruch scheint der »schwarze Protest« auch nicht nur mit Blick auf die Anzahl der Teilnehmenden, die sich offen zu ihren Ansichten zum Thema Schwangerschaft bekannten, gewesen zu sein. Er war auch ein Wendepunkt im Hinblick auf den Diskurs über Abtreibung. Bislang wurde über Abtreibung vor allem aus der Perspektive der individuellen Entscheidung der Frau über ihren Körper gesprochen. »Mein Körper - meine Entscheidung «, dieses Schlagwort aus der feministischen Bewegung wurde zum Symbol für die Erweiterung des Zugangs zu legaler Abtreibung unter würdigen Bedingungen. Während der Demonstrationen am 3. Oktober stand der Slogan auf etlichen Transparenten. Diesen Slogan nutzten dagegen die Anhänger des vollständigen Abtreibungsverbots mit dem Ziel, die gesamte Frauenbewegung lächerlich zu machen, das Bild von Furien-Feministinnen zu kreieren, die sich nur für sich und ihre Sexualität interessieren, und Argumente einzuführen, die die Erweiterung der Möglichkeiten abzutreiben in Zusammenhang mit »leichtfertigem Verhalten von Frauen« setzen.

Es tauchten aber auch Slogans zum Schutz des Lebens und der Gesundheit auf, die bei Inkrafttreten des Gesetzes von Ordo Iuris gefährdet sein könnten. Die Verlagerung des Schwerpunktes von der selbstbestimmten Wahl auf den Schutz des Lebens war nicht die Idee der Organisatoren des "schwarzen Protestes", sondern ein Ergebnis der Aktivitäten der neuen Welle von Feministinnen in Polen. Das herausragende Motto "Abtreibung zum Schutz des Lebens«, das viele Kontroversen hervorrief, bereitete den Boden für die Organisation der Massendemonstrationen am "schwarzen Montag« vor. Dank dieses Mottos sind sich viele Frauen über die Konsequenzen des aktuell geltenden Rechts bewusst geworden, dessen Folge tatsächlich Todesfälle von Frauen und Kindern in Polen sind.

Zweifellos war der 3. Oktober ein enormer Erfolg der Zivilgesellschaft, aber war er auch ein Erfolg mit Blick auf die Gesetzgebung? Kaum zwei Tage nach dem Streik stimmte der Ausschuss für Gerechtigkeit und Menschenrechte für die gänzliche Ablehnung des Gesetzesentwurfs. Am 6. Oktober sprach sich der Sejm für die Entscheidung der Kommissionsmitglieder aus. Mit Blick auf die bisherigen Erfahrungen, die die Bürgerinnen und Bürger mit Protesten gegen das Regierungshandeln beispielsweise im Zusammenhang mit dem Verfassungstribunal gemacht haben, fällt es schwer zu glauben, dass die Regierung plötzlich begonnen hat, auf die Stimmen der Protestierenden zu hören. Ebenso schwer fällt es, sich des Ein- 
drucks zu erwehren, dass das Gesetzesprojekt von Ordo Iuris der Regierung einen Dienst erwiesen hat. Der vorgeschlagene Entwurf hat die Grenzen vieler Polinnen und Polen so weit überschritten, dass jeder weitere (von PiS bereits angekündigte) Gesetzesentwurf zur Verschärfung des Abtreibungsgesetzes »besser« und akzeptabler erscheinen wird, da er weniger radikal sein wird. Der abgelehnte Gesetzesentwurf kann der Regierung zur schrittweisen Reduzierung des bereits jetzt schon nicht großen Bereichs der Ausnahmen vom Abtreibungsverbot dienen.

\section{Erwartet uns eine neuer "Abtreibungskompromiss «?}

Viele Frauen, die sich seit langem im Kampf für Frauenrechte in Polen engagieren, unterstreichen, dass hier nicht der Krieg gewonnen ist, sondern nur eine Schlacht. Hinzu kommt, dass die Schlacht, die die Polinnen in Zukunft erwartet, möglicherweise noch schwieriger wird. Die Regierungspartei wird mit großer Wahrscheinlichkeit die Drohung des vollständigen Abtreibungsverbots nutzen, um die Novellierung des Gesetzes in Richtung größerer Restriktionen vorzuschlagen, wobei diese angesichts einer drohenden absoluten Illegalisierung des Schwangerschaftsabbruchs ein "neuer Kompromiss« werden könnten. An dem Tag, als der Sejm den Gesetzesentwurf »Abtreibungstopp« ablehnte, kündigte Ministerpräsidentin Beata Szydło an, dass PiS ein breit angelegtes Programm zum Schutz des empfan- genen Lebens erarbeiten wird. Ein erster Bestandteil ist bereits bekannt. Am 3. November fand im Sejm die erste Lesung des Regierungsgesetzesentwurfs »Für das Leben« zur Unterstützung von Schwangeren und Familien statt. Bereits am nächsten Tag wurde das Gesetz verabschiedet. Es sieht unter anderem eine Einmalzahlung in Höhe von 4.000 Zloty (zirka 920 Euro) bei der Geburt eines behinderten oder unheilbar kranken Kindes vor. Ein Familienassistent soll die den Eltern bereitgestellte Hilfe koordinieren. Das Gesetz weckte enorme Kontroversen und rief eine Welle der Kritik und Empörung hervor - nicht nur von Experten, sondern auch von Familien mit behinderten Kindern. Zwei Tage nach Verabschiedung des Gesetzes machte Präsident Andrzej Duda die Marschrichtung weiterer legislativer Arbeiten deutlich: "Was den sogenannten Abtreibungskompromiss betrifft, ist es leider so, dass auch Ärzte sagen, dass er Kinder mit Behinderungen nicht ausreichend schützt, dazu gehören auch Kinder mit Down-Syndrom. Das ist etwas, was ich nicht akzeptieren kann. Wenn mich heute jemand nach dem "Kompromiss" fragt, dann sage ich gerade heraus - von diesem Standpunkt aus ist er nicht ausreichend. Das macht Veränderungen erforderlich [...].«Alles weist darauf hin, dass die polnische Regierung konsequent auf einen neuen "Kompromiss" hinarbeiten wird. Es bleibt die Frage, was die polnischen Frauen dazu sagen werden.

Übersetzung aus dem Polnischen: Silke Plate

Über die Autorin

Małgorzata Druciarek, Soziologin, leitet das Observatorium zur Geschlechtergleichheit des Instituts für Öffentliche Angelegenheiten (Instytut Spraw Publicznych - ISP) in Warschau. Ihre Forschungsschwerpunkte sind die Gleichberechtigung von Männern und Frauen und die Familienpolitik. 


\section{Umfragen zur Abtreibungsdebatte}

Grafik 1: Sollte das Gesetz zum Schwangerschaftsabbruch geändert werden? Sollte es ... (\%)

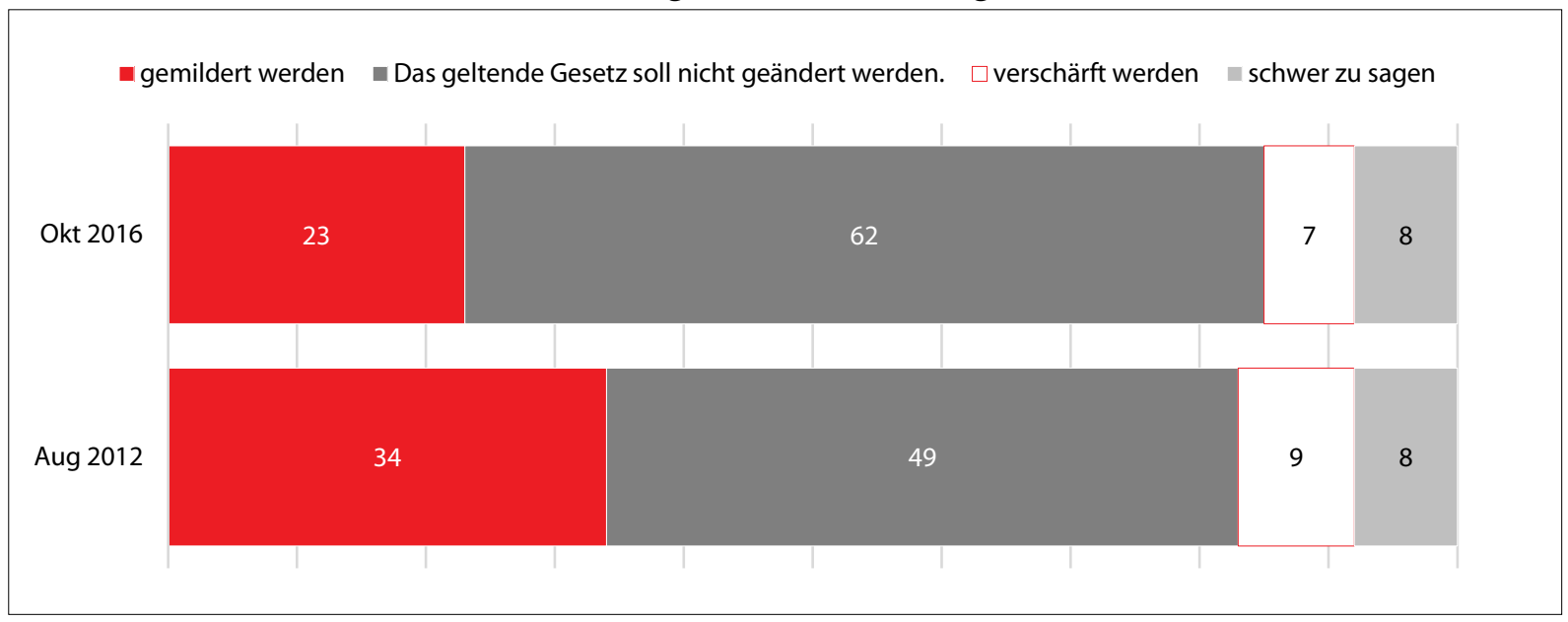

Quelle: CBOS Komunikat z badań Nr 144/2016: Jakiego prawa aborcyjnego oczekuja Polacy? [Welches Abtreibungsgesetz erwarten die Polen?] Warszawa 10/2016. <www.cbos.pl>

Grafik 2: Die Unterstützung des »schwarzen Protestes« und des Landesweiten Streiks der Frauen

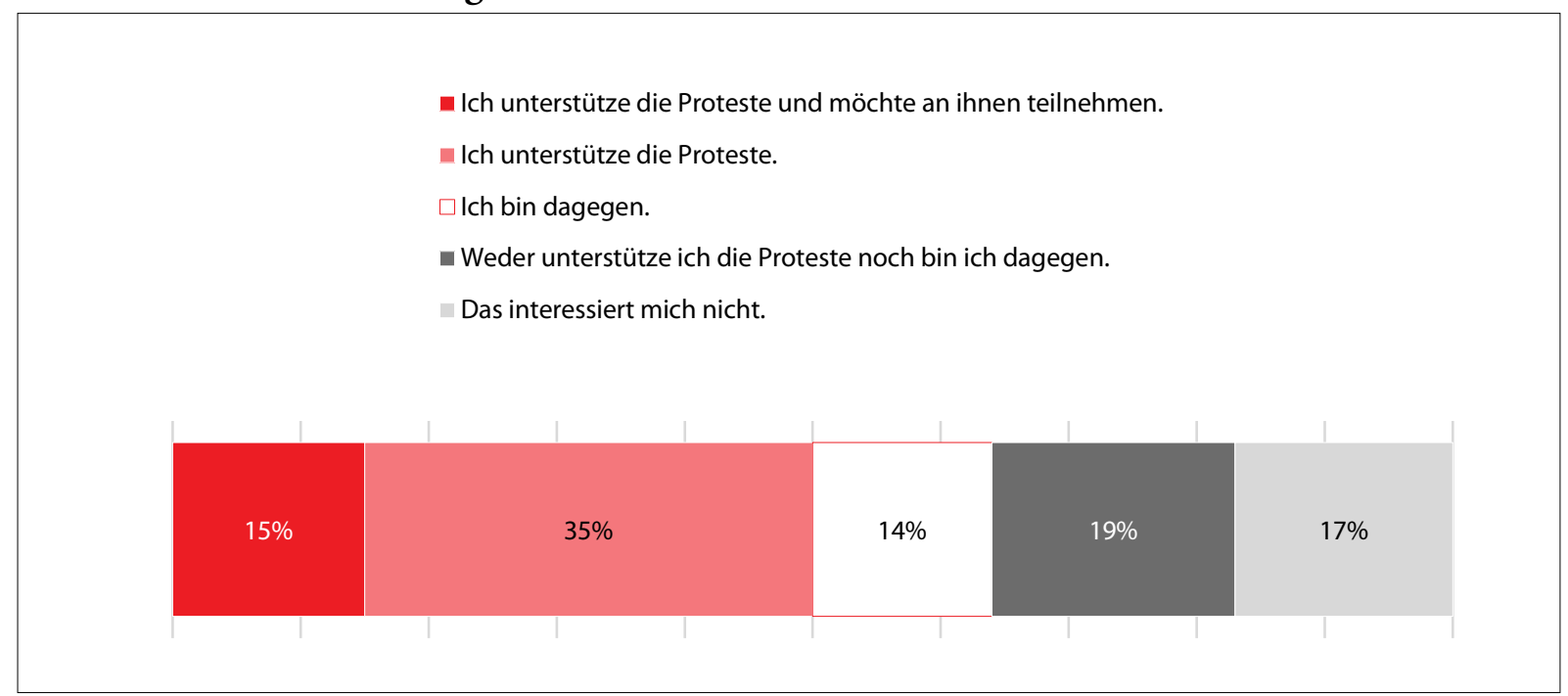

Umfrage des Meinungsforschungsinstituts IPSOS für OKO.press, 28.-30.09.2016, <https://oko.press/bunt-kobiet-stal-sie-masowypolowa-popiera-jedna-siodma-przeciw/> (abgerufen am 15.11.2016) 


\begin{tabular}{|c|c|c|c|c|c|c|c|c|c|}
\hline & $\stackrel{5}{5}$ & $\curvearrowright$ & 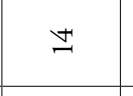 & $\Xi$ & $\stackrel{\curvearrowleft}{\sim}$ & $\mathbb{N}$ & $\stackrel{\star}{N}$ & $\stackrel{\infty}{\wedge}$ & \\
\hline & ב. & $\triangleright$ & 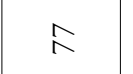 & $\curvearrowright$ & 8 & خे & $\approx$ & 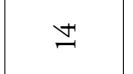 & \\
\hline & $\stackrel{5}{S}$ & $=$ & $\stackrel{\infty}{\sim}$ & $\stackrel{\varrho}{-}$ & $\stackrel{m}{ }$ & $\Re$ & 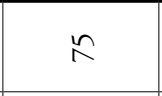 & $\gtrless$ & \\
\hline & t. & $\triangleright$ & $\nabla$ & $\Re$ & 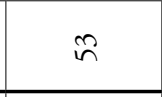 & 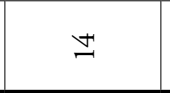 & 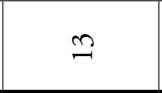 & 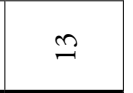 & \\
\hline & $\vec{v}$ & $=$ & $\stackrel{\infty}{\sim}$ & $\stackrel{m}{=}$ & 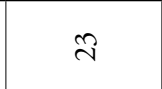 & $\Re$ & $\stackrel{+}{N}$ & $\Re$ & \\
\hline & 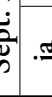 & $\bar{\infty}$ & $\nabla$ & $\stackrel{\infty}{\curvearrowright}$ & $\vec{\sigma}$ & $\stackrel{\bullet}{\circ}$ & 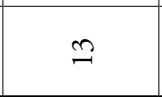 & 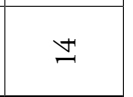 & \\
\hline & 5 & $\wedge$ & 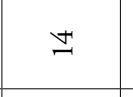 & $\stackrel{m}{2}$ & $\hat{\sim}$ & $\approx$ & $\hat{\sigma}$ & $\Re$ & \\
\hline & . & $\hat{\infty}$ & $\curvearrowright$ & $\stackrel{\infty}{\wedge}$ & $\hat{n}$ & $\stackrel{\sim}{\sim}$ & $\vec{\sim}$ & 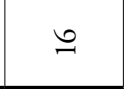 & \\
\hline & $\vec{v}$ & $\wedge$ & $\Xi$ & $\simeq$ & $\approx$ & $\tilde{\sigma}$ & $\pi$ & $\stackrel{\Re}{\wedge}$ & \\
\hline & $\approx$ & $\infty$ & $\stackrel{\infty}{\unrhd}$ & $\stackrel{\infty}{\wedge}$ & 8 & $\stackrel{\sim}{\sim}$ & $\ddot{\sim}$ & $\stackrel{\infty}{\sim}$ & \\
\hline & $\vec{v}$ & $n$ & $a$ & $\simeq$ & $\vec{\sim}$ & $\approx$ & 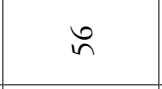 & ๖ & \\
\hline & $\begin{array}{l}\dot{b} \\
\dot{b}\end{array}$ & $\bar{\sigma}$ & $\mathscr{\infty}$ & $\curvearrowright$ & 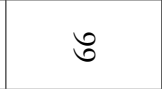 & $\ddot{m}$ & ஜे & $\ddot{\sim}$ & \\
\hline & 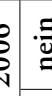 & $\infty$ & $\cong$ & $\stackrel{\circ}{\sim}$ & $\stackrel{\sim}{\sim}$ & $\check{n}$ & $\overleftarrow{J}^{+}$ & $\mathbb{N}$ & \\
\hline & 它 & $\triangleright$ & $\curvearrowright$ & $\stackrel{\curvearrowright}{\wedge}$ & $\widetilde{\sigma}$ & $\hat{\lambda}$ & $\vec{\lambda}$ & $\stackrel{\circ}{\circ}$ & \\
\hline & $\hat{v}$ & $\infty$ & $\Xi$ & $\cong$ & $\tilde{\lambda}$ & $\stackrel{\sim}{\forall}$ & $\vec{n}$ & 8 & \\
\hline & . & $\infty$ & $\triangleright$ & $\curvearrowright$ & Ъ & $\underset{F}{\sim}$ & $\stackrel{r}{\circ}$ & $\stackrel{\infty}{\sim}$ & \\
\hline & $\vec{v}$ & $\infty$ & 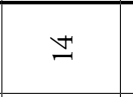 & $\cong$ & $\vec{\lambda}$ & $\underset{\forall}{*}$ & fे & $\stackrel{\infty}{n}$ & \\
\hline &. & 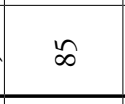 & $\curvearrowright$ & $\stackrel{\curvearrowright}{\curvearrowright}$ & $\approx$ & $\underset{\forall}{\forall}$ & $\stackrel{\infty}{\infty}$ & $\stackrel{\infty}{\sim}$ & \\
\hline & 5 & $\bullet$ & $\Xi$ & $\stackrel{\circ}{\sim}$ & $\stackrel{\sim}{\sim}$ & 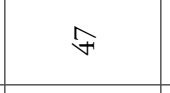 & ' & $\stackrel{\infty}{n}$ & \\
\hline & 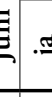 & $\triangleright$ & $\hat{\wedge}$ & $\mathbb{N}$ & $\sigma$ & $\stackrel{\infty}{m}$ & ' & $\hat{\imath}$ & \\
\hline & 5 & $\bullet$ & $=$ & 으 & $\curvearrowleft$ & ळે & ' & ' & \\
\hline & 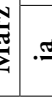 & $\infty$ & $\widetilde{\infty}$ & $\infty$ & $\nabla$ & † & ' & ' & \\
\hline & & 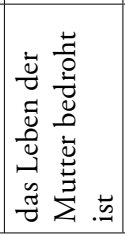 & 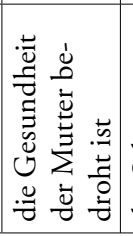 & 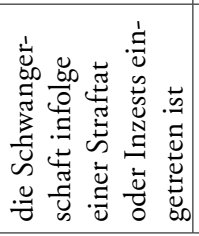 & 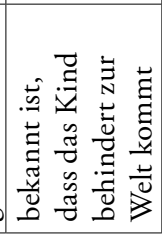 & 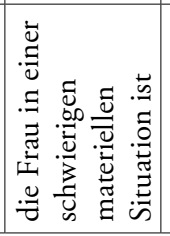 & 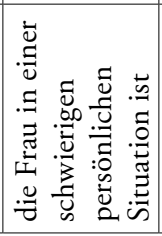 & 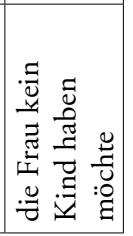 & $\dddot{\Sigma}$ \\
\hline
\end{tabular}




\section{1. - 14. November 2016}

\begin{tabular}{|c|c|}
\hline 01.11 .2016 & $\begin{array}{l}\text { In einem gemeinsamen Fernsehinterview warnen Włodzimierz Czarzasty, Parteivorsitzender der Demokratischen } \\
\text { Linksallianz (Sojusz Lewicy Demokratyzcnej - SLD), und Janusz Piechociński, Mitglied des Parteirates der Pol- } \\
\text { nischen Bauernpartei (Polskie Stronnictow Ludowe - PSL) und ehemaliger Wirtschaftsminister, vor einer zuneh- } \\
\text { menden Spaltung der polnischen Gesellschaft angesichts der von der Landesstaatsanwaltschaft in der vergange- } \\
\text { nen Woche angeordneten Exhumierung der Opfer des Flugzeugunglücks von Smolensk (2010). Das Prinzip der } \\
\text { Regierungspartei Recht und Gerechtigkeit (Prawo i Sprawiedliwość - PiS) sei es, Konflikte zu ihrem Vorteil zu } \\
\text { schüren. Piechociński sagt, im konkreten Fall handele es sich um eine Abrechnung mit den damaligen Politi- } \\
\text { kern und Untersuchungsbefugten der polnischen Staatsanwaltschaft. Er sprach sich für eine Exhumierung der } \\
\text { Todesopfer nur mit Zustimmung der Angehörigen aus. }\end{array}$ \\
\hline 02.11 .2016 & $\begin{array}{l}\text { Der Gesetzesentwurf der Regierung »Für das Leben« wird an den Sejm geleitet. Er sieht Unterstützung für Fami- } \\
\text { lien mit unheilbar kranken oder behinderten Neugeborenen vor, u. a. eine Einmalzahlung von } 4.000 \text { Zloty } \\
\text { (ca. } 920 \text { Euro). }\end{array}$ \\
\hline 03.11 .2016 & $\begin{array}{l}\text { In einem Radiointerview spricht sich der Parteivorsitzende von Die Moderne (Nowoczesna), Ryszard Petru, für } \\
\text { die Einheit innerhalb der Opposition in fundamentalen Angelegenheiten aus, beispielsweise beim Konflikt um } \\
\text { das Verfassungstribunal oder bei Fragen der Freiheit der Bürger. Darüber hinaus seien die Parteien unterschied- } \\
\text { lich und stünden in einem Konkurrenzverhältnis. Dieses aufzuheben sei bei bevorstehenden Wahlen aus Grün- } \\
\text { den der Staatsräson möglich. }\end{array}$ \\
\hline 04.11 .2016 & $\begin{array}{l}\text { In den polnischen Medien wird über die Reaktion der polnischen Regierung auf die Empfehlungen der Euro- } \\
\text { päischen Kommission im Zusammenhang mit der Reform des Verfassungstribunals berichtet. Die Kommission } \\
\text { hatte eine "systembedingte Gefahr für den Rechtsstaat« in Polen festgestellt. Aus der Stellungnahme der Regie- } \\
\text { rung wird u. a. zitiert, dass die Empfehlungen nicht umgesetzt werden können, da dadurch die polnische Ver- } \\
\text { fassung und Gesetzgebung verletzt werden würden. Die Kommission gehe von der These von der grundlegen- } \\
\text { den Bedeutung des Verfassungstribunals für das polnische Rechtssystem aus, dies sei jedoch nicht zutreffend, } \\
\text { da auch das Staatstribunal und der Präsident konstitutionelle Kompetenzen hätten. Darüber hinaus sei ein Teil } \\
\text { der Empfehlungen infolge des neuen Gesetzes zum Verfassungstribunal vom 22. Juli bedeutungslos geworden. }\end{array}$ \\
\hline 05.11 .2016 & $\begin{array}{l}\text { Das Außenministerium distanziert sich von den Empfehlungen des Ausschusses für Menschenrechte der Ver- } \\
\text { einten Nationen, die er aufgrund seiner Beunruhigung über die Lage in Polen hinsichtlich des Konflikts um das } \\
\text { Verfassungstribunals, das Abtreibungsgesetz und die Reform der öffentlichen Medien geäußert hat. Es sei enttäu- } \\
\text { schend, dass die in umfangreichen Erläuterungen und einem intensiven Dialogs vermittelten Informationen nicht } \\
\text { aufVerständnis vonseiten des Ausschusses gestoßen seien, heißt es in der Stellungnahme des Außenministeriums. }\end{array}$ \\
\hline 06.11 .2016 & $\begin{array}{l}\text { Als erster Präsident der Republik Polen hält sich Andrzej Duda zu einem offiziellen Besuch in Jordanien auf. Jor- } \\
\text { danien sei das Land im Nahen Osten, das der Europäischen Union und der NATO am nächsten stehe. Es zeichne } \\
\text { sich durch Stabilität und die Achtung demokratischer Prinzipien aus. Duda äußerte die Hoffnung auf weitere } \\
\text { bilaterale Zusammenarbeit im wirtschaftlichen und militärischen Bereich. In Amman eröffnet er eine gemein- } \\
\text { same Veranstaltung zur Zusammenarbeit der Rüstungsindustrie. }\end{array}$ \\
\hline 07.11 .2016 & $\begin{array}{l}\text { Maciej Konieczny, Agnieszka Dziemianowicz-Bąk und Adrian Zandberg, Vorstandsmitglieder der Partei Gemein- } \\
\text { sam (Razem), werfen Recht und Gerechtigkeit (Prawo i Sprawiedliwość-PiS) vor, rechtsradikale Gruppierungen } \\
\text { wie das National-Radikale Lager (Obóz Narodowo-Radykalny - ONR) finanziell, materiell und ideell zu unter- } \\
\text { stützen. Razem könne aus den vergangenen Wochen } 93 \text { Beispiele anführen. Insbesondere ONR sei eine Orga- } \\
\text { nisation, die die Demokratie in Polen zerstören wolle. Razem fordert Aufklärung über das Ausmaß der Zusam- } \\
\text { menarbeit und die personelle Beteiligung von PiS. }\end{array}$ \\
\hline 8.11 .2016 & $\begin{array}{l}\text { Außenminister Witold Waszczykowski nimmt in Bukarest (Rumänien) an einem Außenministertreffen der } \\
\text { Länder der NATO-Ostflanke (Bulgarien, Estland, Lettland, Litauen, Polen, Rumänien, Slowakei, Tschechien, } \\
\text { Ungarn) und der stellvertretenden NATO-Generalsekretärin Rose Gottemoeller teil. Die Minister unterstrei- } \\
\text { chen ihre Einheit und Solidarität angesichts der aggressiven Aktivitäten Russlands. Waszczykowski betont, das } \\
\text { Forum diene nicht dazu, die NATO zu spalten, sondern um Fragen der Sicherheit in den ostmitteleuropäischen } \\
\text { Ländern Nachdruck zu verleihen. }\end{array}$ \\
\hline 09.11 .2016 & $\begin{array}{l}\text { Präsident Andrzej Duda gratuliert dem US-amerikanischen republikanischen Präsidentschaftskandidaten Donald } \\
\text { Trump in einem Brief zu seinem Wahlsieg. Darin hebt Duda den Erfolg des NATO-Gipfels im Juli in War- } \\
\text { schau hervor, bei dem die USA sich dazu verpflichteten, ihre militärische Präsenz in Polen zu verstärken. Weiter } \\
\text { bedankt er sich bei Trump für das Treffen mit Angehörigen der Polonia während des Wahlkampfes und für die } \\
\text { positiven Äußerungen über Polen und die Rolle der Polen in der Geschichte der USA. }\end{array}$ \\
\hline
\end{tabular}




\begin{tabular}{|c|c|}
\hline 09.11 .2016 & $\begin{array}{l}\text { Außenminister Witold Waszczykowski bewertet den Sieg des Republikaners und politischen Quereinsteigers } \\
\text { Donald Trump bei der US-Präsidentschaftswahl als Warnung an die politischen Eliten in Europa. Es müssen } \\
\text { ernsthafte Korrekturen in der europäischen Politik vorgenommen werden. Das Wahlergebnis zeige, dass ein } \\
\text { Teil der US-Amerikaner zu Werten und Traditionen zurückkehren wolle. In Zeiten der Globalisierung stehe der } \\
\text { Durchschnittsbürger vor vielen Entscheidungen, die sein Leben beträfen, und suche Führer, die ihm helfen, so } \\
\text { Waszczykowski. }\end{array}$ \\
\hline 10.11.2016 & $\begin{array}{l}\text { Präsident Andrzej Duda unterzeichnet das Gesetz zur Unterstützung von Schwangeren und Familien "Für das } \\
\text { Leben«. Demnach werden Hilfsleistungen für Familien mit einem unheilbar kranken oder behinderten Neuge- } \\
\text { borenen von einem Familienassistenten koordiniert und wird eine Einmalzahlung von } 4.000 \text { Zloty (ca. } 920 \text { Euro) } \\
\text { ausgezahlt. Das Gesetz tritt am 1. Januar } 2017 \text { in Kraft. }\end{array}$ \\
\hline 11.11 .2016 & $\begin{array}{l}\text { Am Nationalfeiertag der Unabhängigkeit (1918) finden in Warschau außer den offiziellen Feierlichkeiten mit } \\
\text { den höchsten Staatsvertretern drei Demonstrationszüge statt. Nach Angaben der Polizei nahmen am Marsch der } \\
\text { Unabhängigkeit, organisiert von rechten und rechtsradikalen Gruppierungen (National-Radikales Lager/Obóz } \\
\text { Narodowo-Radykalny - ONR u. a.), ca. } 75.000 \text { Teilnehmer, an der Demonstration des Komitees zur Verteidi- } \\
\text { gung der Demokratie (Komitet Obrony Demokracji - KOD) ca. } 10.000 \text { und am Marsch aus dem antifaschisti- } \\
\text { schen Umfeld ca. } 900 \text { Personen teil. Die Demonstrationen verliefen ohne größere Zwischenfälle. }\end{array}$ \\
\hline 11.11 .2016 & $\begin{array}{l}\text { Aus Anlass des Nationalfeiertags der Unabhängigkeit (1918) fordert der Parteivorsitzende von Recht und Gerech- } \\
\text { tigkeit (Prawo i Sprawiedliwość - PiS), Jarosław Kaczyński, in Krakau die beständige Verteidigung von Frei- } \\
\text { heit und Unabhängigkeit. Es gebe immer mehr Akteure, die die Freiheit und Souveränität Polens und das Recht } \\
\text { Polens auf Selbstbestimmung untergrüben. Die einzige Antwort darauf sei, die eigenen Kräfte auszubauen, was } \\
\text { für das gesamte Spektrum von der Armee bis zur Kultur gelten müsse. PiS reiche über alle Unterschiede hinweg } \\
\text { allen die Hand, die für ein starkes Polen und Europa sind. }\end{array}$ \\
\hline 13.11 .2016 & $\begin{array}{l}\text { In Brüssel treffen sich die EU-Außenminister, um über die Folgen des Ausgangs der US-Präsidentenwahl zu bera- } \\
\text { ten, die der Republikaner Donald Trump Anfang der Woche gewann. Außenminister Witold Waszczykowski } \\
\text { sagt am Rande des informellen Treffens, dass die EU viele Probleme zu lösen habe, jedoch sei die Sorge um den } \\
\text { US-Wahlausgang übertrieben. Des Weiteren spricht er sich dafür aus, dass die EU in der Verteidigungspolitik } \\
\text { als Einheit auf Gefahren reagieren sollte. }\end{array}$ \\
\hline 14.11 .2016 & $\begin{array}{l}\text { Verteidigungsminister Antoni Macierewicz tritt Einschätzungen entgegen, dass der Aufbau der paramilitäri- } \\
\text { schen Militäreinheiten zur Territorialen Verteidigung (Wojska Obrony Terytorialnej - WOT) die Finanzen für } \\
\text { die Modernisierung der polnischen Streitkräfte reduzieren könnten. Die WOT seien die kostengünstigste Mög- } \\
\text { lichkeit, die Armee und ihre Verteidigungskapazitäten zu vergrößern, so Macierewicz. Die WOT sollen zur mili- } \\
\text { tärischen Verteidigung sowie bei Antikrisen- und Antiterrormaßnahmen eingesetzt werden. Bis } 2019 \text { sollen sie } \\
53.000 \text { Soldaten umfassen, für diese Aufbauphase würden 3,6 Mrd. Zloty (ca. } 815 \text { Mio. Euro) bereitgestellt. }\end{array}$ \\
\hline
\end{tabular}

Sie können die gesamte Chronik seit 2007 auch auf <http://www.laender-analysen.de/polen/> unter dem Link "Chronik« lesen. 


\section{ÜBER DIE POLEN-ANALYSEN}

Die Polen-Analysen erscheinen zweimal monatlich als E-Mail-Dienst. Sie werden gemeinsam vom Deutschen PolenInstitut Darmstadt, der Bremer Forschungsstelle Osteuropa und der Deutschen Gesellschaft für Osteuropakunde herausgegeben.

Ein Archiv der Polen-Analysen finden Sie im Internet unter <www.laender-analysen.de/polen>

Kostenloses Abonnement unter <http://www.deutsches-polen-institut.de/Newsletter/subscribe.php >

Diese Analysen finden Sie online als Lizenzausgabe auf

$<$ bpb.de>

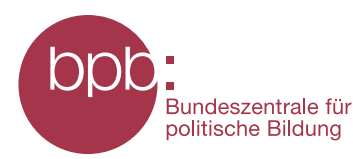

Deutsches Polen-Institut Darmstadt (<www.deutsches-polen-institut.de $>$ )

Das seit 1980 tätige Deutsche Polen-Institut Darmstadt (DPI) ist ein Forschungs-, Informations- und Veranstaltungszentrum für polnische Kultur, Geschichte, Politik, Gesellschaft und die deutsch-polnischen Beziehungen, die sich im Kontext der europäischen Integration entwickeln. Institutionelle Träger des DPI sind das Land Hessen, die Kultusminister der Länder, das Auswärtige Amt und die Wissenschaftsstadt Darmstadt. Einen wesentlichen Beitrag zur Verwirklichung der Institutsziele leisten private Stiftungen. Ziel der Vermittlertätigkeit des DPI ist es, »die zu interessieren, auf die es politisch, wirtschaftlich, gesellschaftlich und kulturell im deutsch-polnischen Verhältnis ankommt« (Leitlinien 1997). Es geht um die Entscheider und Multiplikatoren in Politik, Kultur, Bildung, Verwaltung, Medien und Wirtschaft. Das DPI versteht sich in Kooperation mit den Orten wissenschaftlicher Polen-Kompetenz an deutschen Hochschulen und Forschungsinstituten als verbindendes und vernetzendes Zentrum. Mit der über 68.000 Bände zählenden multidisziplinären Fachbibliothek für Polen, die eine einzigartige Sammlung polnischer Belletristik in der Originalsprache und in deutscher Übersetzung umfasst, ist das DPI ein geschätzter Ort der Recherche und des wissenschaftlichen Arbeitens.

Forschungsstelle Osteuropa an der Universität Bremen (<www.forschungsstelle.uni-bremen.de>)

1982 gegründet, widmet sich die Forschungsstelle Osteuropa an der Universität Bremen der interdisziplinären Analyse der Länder Ost- und Ostmitteleuropas in Zeitgeschichte und Gegenwart. Der Forschungsschwerpunkt liegt dabei auf der Rolle von "Dissens und Konsens«, von Opposition und Zivilgesellschaft in ihrem historischen, politischen, gesellschaftlichen und kulturellen Kontext. Die Forschungsstelle besitzt in ihrem Archiv eine einzigartige Sammlung alternativer Kulturgüter und unabhängiger Texte aus den ehemaligen sozialistischen Ländern. Darunter befindet sich auch eine umfangreiche Sammlung des "Zweiten Umlaufs«, die das Schrifttum und Dokumente unabhängiger Initiativen und gesellschaftlicher Gruppen in Polen aus der Zeit von 1976 bis zum Umbruch umfasst. Hinzu kommt eine umfangreiche Bibliothek mit wissenschaftlicher Literatur. Mit Archiv, Bibliothek und zwei wissenschaftlichen Abteilungen ist die Forschungsstelle auch eine Anlaufstelle sowohl für Gastwissenschaftler als auch für die interessierte Öffentlichkeit.

Eine der Hauptaufgaben der Forschungsstelle ist die Information der interessierten Öffentlichkeit. Dazu gehören unter anderem regelmäßige E-Mail-Informationsdienste für Politik, Wirtschaft, Zivilgesellschaft und Medien.

Das Institut für Öffentliche Angelegenheiten (Instytut Spraw Publicznych - ISP) in Warschau ist einer der führenden Think Tanks in Polen und seit 1995 als unabhängiges Forschungszentrum zu grundlegenden Fragen des öffentlichen Lebens tätig. Das ISP kooperiert eng mit zahlreichen Experten und Forschern wissenschaftlicher Einrichtungen im In- und Ausland. $<$ www.isp.org.pl>

Herausgegeben mit finanzieller Unterstützung der Stiftung für deutsch-polnische Zusammenarbeit

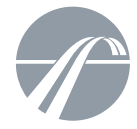

FUNDACJA WSPÓtPRACY
POLSKO-NIEMIECKIEJ

POLSKO-NIE
STIFTUNG

STIFTUNG

FUR DEUTSCH-POLNISC

Die Meinungen, die in den Polen-Analysen geäußert werden, geben ausschließlich die Auffassung der Autoren wieder

Abdruck und sonstige publizistische Nutzung sind nach Rücksprache mit der Redaktion gestattet.

Redaktion: Prof. Dr. Dieter Bingen (verantwortlich) (Darmstadt), Silke Plate M.A. (Bremen) Technische Gestaltung: Matthias Neumann

Polen-Analysen-Layout: Cengiz Kibaroglu, Matthias Neumann

Alle Ausgaben der Polen-Analysen sind mit Themen- und Autorenindex archiviert unter www.laender-analysen.de

Die Polen-Analysen werden im Rahmen der Datenbank World Affairs Online (WAO) ausgewertet und sind im Portal IREON www.ireon-portal.de recherchierbar

ISSN 1863-9712 @ 2016 by Deutsches Polen-Institut Darmstadt und Forschungsstelle Osteuropa, Bremen

Kontakt: Dr. Andrzej Kaluza, Presse- und Öffentlichkeitsarbeit, Deutsches Polen-Institut, Residenzschloss, Marktplatz 15,

D-64283 Darmstadt, Tel.: +49/6151/4202-20, Fax: +49/6151/4202-10, E-Mail: polen-analysen@dpi-da.de, Internet: www.laender-analysen.de/polen 